\title{
Sur les traces des Maures, entre sable et poussière
}

Following the tracks of the Moors, between sand and dust

\section{Catherine Taine-Cheikh}

\section{(2) OpenEdition}

Journals

Édition électronique

URL : https://journals.openedition.org/tc/7304

DOI : $10.4000 /$ tc. 7304

ISSN : 1952-420X

Éditeur

Éditions de l'EHESS

\section{Édition imprimée}

Date de publication : 15 décembre 2013

Pagination : 188-209

ISBN : 978-2-7351-1654-6

ISSN : 0248-6016

\section{Référence électronique}

Catherine Taine-Cheikh, « Sur les traces des Maures, entre sable et poussière », Techniques \& Culture [En ligne], 61 | 2013, mis en ligne le 15 décembre 2016, consulté le 29 septembre 2022. URL : http:// journals.openedition.org/tc/7304; DOI : https://doi.org/10.4000/tc.7304 


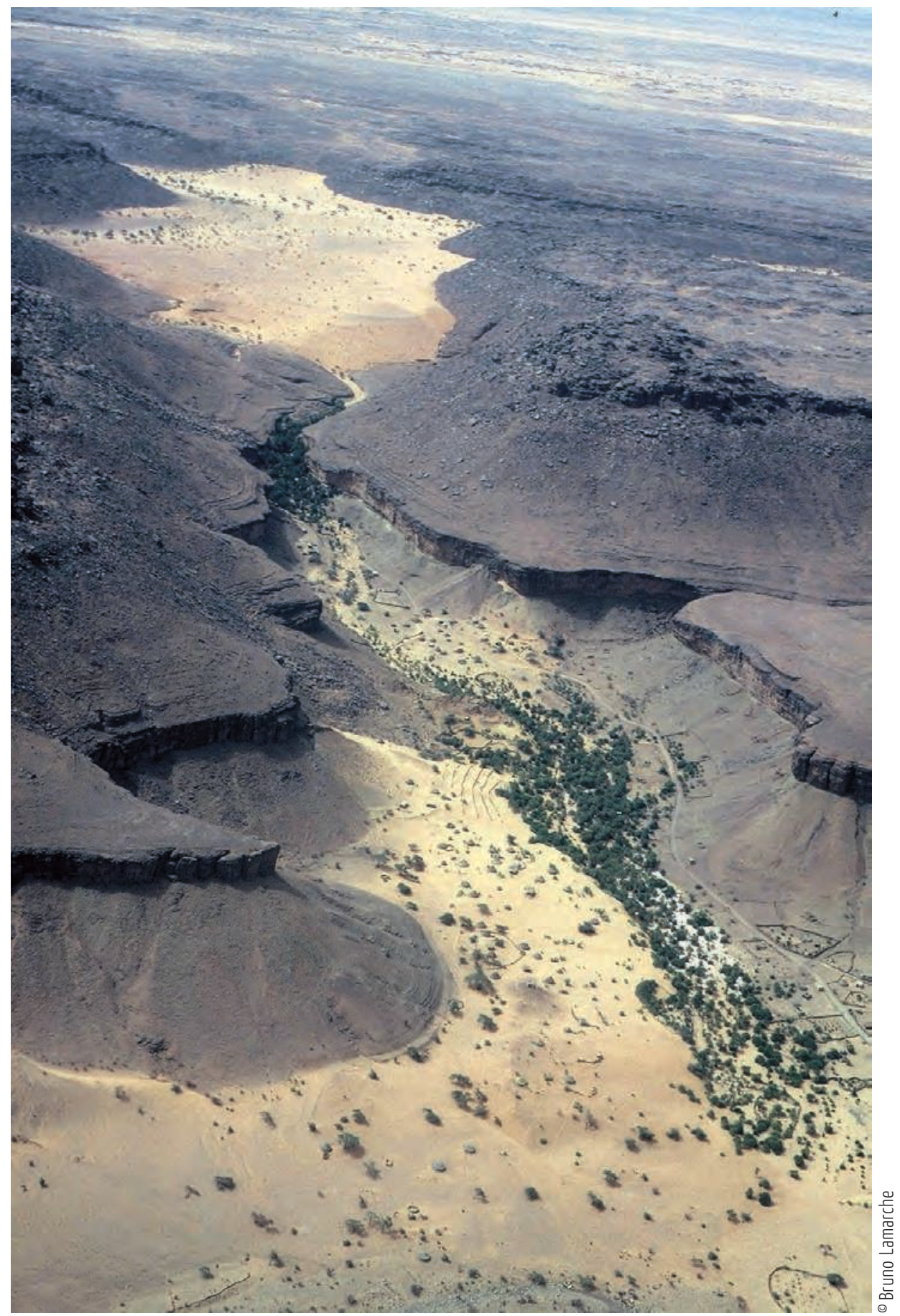


Catherine Taine-Cheikh

LACITO (CNRS-Université Paris III Sorbonne Nouvelle) cath.tainecheikh@gmail.com
Vivre le sable! Corps, matière et sociétés - 3

Techniques \& Culture 61, 2013/2: 188-209

\section{SUR LES TRACES DES MAURES, ENTRE SABLE ET POUSSIËRE}

Pour une partie des sept milliards d'humains, habitants de mégapoles surpeuplées, le désert est devenu par contraste un lieu attractif et, s'il existe toutes sortes de désert, ceux de sable semblent particulièrement aptes à fasciner les imaginations. On peut avoir, sur cet élément, de multiples représentations, mais il est certain qu'entre le bac à sable des bambins - ou même la « Mer de Sable » du parc d'attractions d'Ermenonville - et les 900 kilomètres désertiques de la Majabât el Koubra parcourue par Théodore Monod, il n’y a pas de commune mesure. M'intéressant aux populations nomades maures (les Bị̣̂̂an) qui (sur)vivent dans les régions désertiques et semi-désertiques d'Afrique du Nord-Ouest, je me propose d'explorer leurs représentations du désert saharien, et plus particulièrement du sable, à travers les ressources linguistiques et littéraires de leur dialecte, le hasssāniyya ${ }^{1}$.

Dans la première partie, l'accent sera mis sur les caractéristiques des terrains à sable. Dans la seconde, j'envisagerai les formes de vie se manifestant dans cet univers et étudierai l'interaction des hommes avec le milieu.

\section{Les paysages de sable}

Le Sahara abonde en terrains sablonneux, mais il ne se réduit pas à cela, car il faut compter aussi, dans les zones arides, avec les reliefs rocheux, les regs - surfaces 
caillouteuses ou graveleuses - et les dépressions salines ou argileuses. Au total, les sables n'occuperaient que « 20 \% des surfaces au Sahara» (Monod 1988: 63), tout en en constituant la caractéristique majeure. Ceci pourrait expliquer les glissements de sens que l'on observe dans le lexique maure.

\section{El Ghallaouiya (vers le nord)}

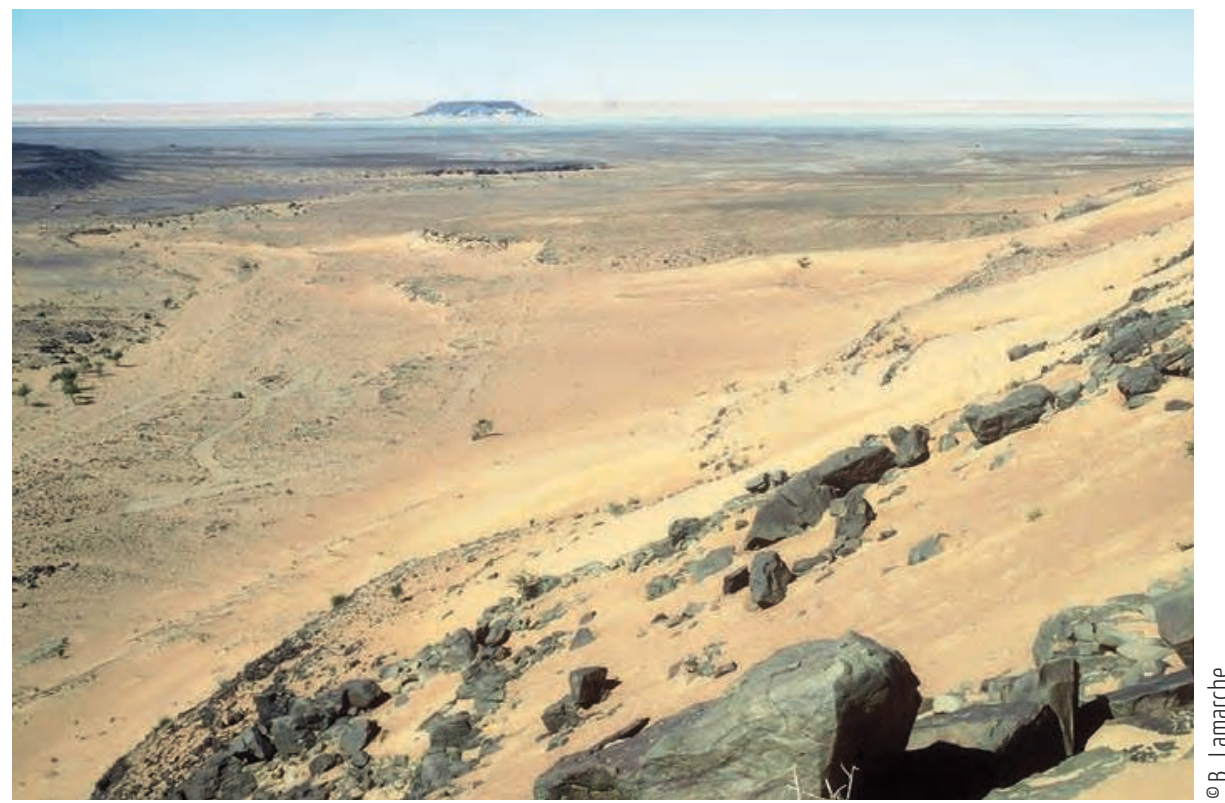

\section{Le sable et la terre}

En arabe littéraire, le terme général pour « sable » est raml tandis que le féminin ramla désigne « un morceau de terrain sablonneux » (Kazimirski 1860 : I, 928). En ḥassāniyya, la forme usitée ( ramlä) est plus proche de la seconde et elle cumule le sens de «sable, poussière » avec celui de « terrain sablonneux, "farineux" ». Dans la seconde acception, on peut également entendre une expression plus littérale (trâa mrramlyä) où la forme participiale mramli, de même base que ramlä, détermine le substantif (de genre féminin) trâb « terre, terrain ».

Chez les Maures, les termes de la racine $\mathrm{rm} l$ renvoient toujours à une nature particulière de sable: "sable blanc, pulvérulent, très fluide, que l'on rencontre dans de nombreux puits de l'Azawād et qui s'effrite au moindre contact comme de l'eau », selon la définition de Poussibet, bon connaisseur de la région de Tombouctou (1962).

Il ne s'agit pas pour autant de termes très usités, car les hassanophones leur préfèrent de beaucoup l'expression plus imagée de at-trâb al-bäyđạa (litt. « la terre blanche »), voire celle, plus elliptique mais souvent équivalente en contexte, de trâab. En effet, comme son équivalent classique turāb, le lexème trấb signifie non seulement « terre, sol, terrain », mais également " poussière », et c'est sans doute de là que lui vient son aptitude à désigner assez communément le sable, notamment sous son aspect poudreux. 
at-trâab al-bäyđạa « le terrain aréneux et découvert, la région de dunes » prend tout son sens par opposition à at-trâab al-kaḥlä (litt. « la — noire ») « la région de montagne ». Par contre, on emploie, dans des sens assez voisins, trâab șavrạa (litt. « — jaune ») pour « terre desséchée, dénudée », trấb zagimä (litt. «— fauve clair ») pour « terrain sablonneux, uni, sans relief notable » et trâb hamra (litt. «— rouge ») pour « terrain aride, brûlé par le soleil, sans pâturage » (souvent " terrain sablonneux, à gros grains rouges », mais pas nécessairement). Bien que les couleurs du sable varient beaucoup, du presque noir au presque blanc, en passant par différentes nuances du jaune, de l'orangé et même du vert, l'emploi des adjectifs de couleurs pour dénoter les caractéristiques géophysiques dominantes du sol ou sa couverture végétale est donc en partie symbolique.

Il faut noter par ailleurs que la nature du sable tend à changer avec la couleur et que le sable à plus gros grain, de couleur généralement plus soutenue, ne s'appellera ni ramlä ni at-trâab al-bäyđ̛̣a, mais ḥaṣbä ou ḥsii. En arabe littéraire, les collectifs haṣba et hașan signifient « cailloux », et même s'il n'est pas rare de retrouver leurs équivalents en dialectal, c'est généralement avec le sens de « petits cailloux, gravillons », plus exceptionnellement, avec celui de « (grains de) sable ». Pourtant, c'est bien ce sens que prend couramment le collectif haṣbä en hassāniyya, comme l'atteste la tournure ši mlân mn ol-haṣää qui se dit

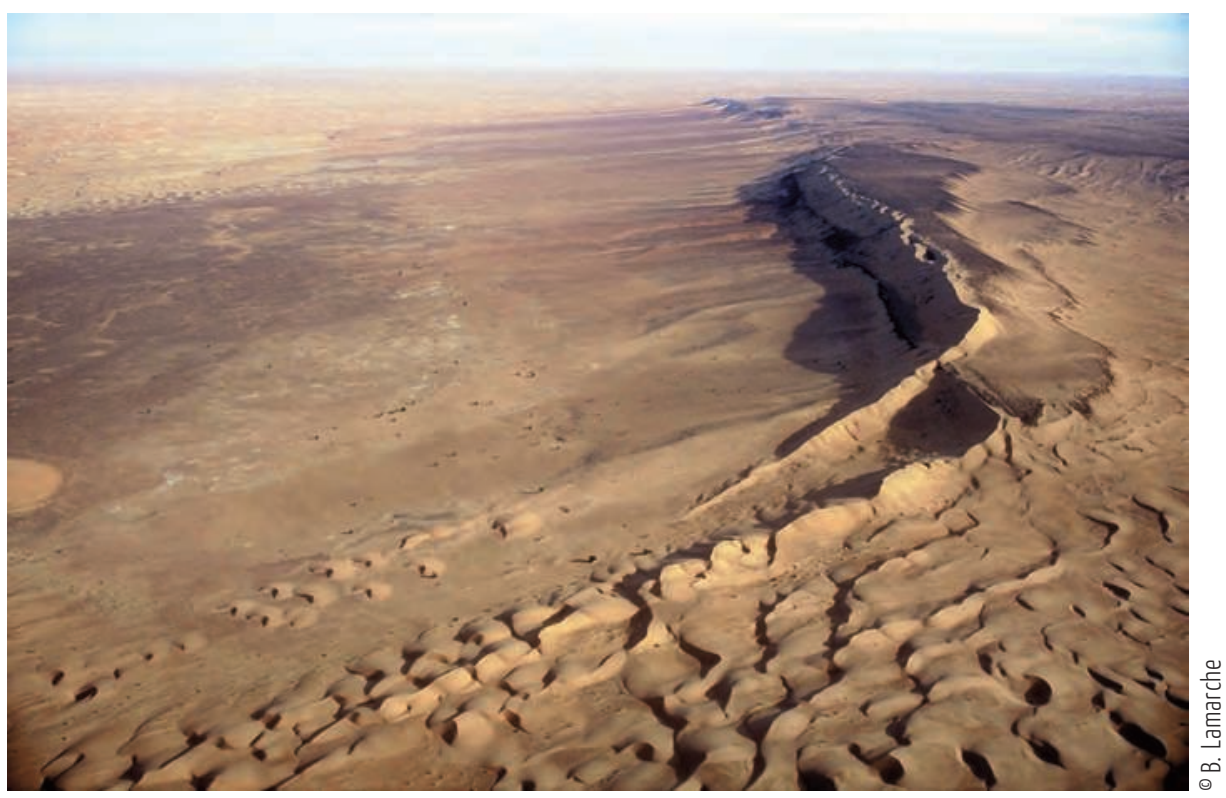

El Bäyyəọ (vers le nord)

d'une chose (viande par exemple) pleine de sable. Quant à son synonyme hsi (nom d'unité hasyä), qui appartient à la même racine que le verbe hṣa yohṣi « dénombrer quelque chose en grande quantité », il est resté - malgré le glissement de sens - emblématique de la plus petite chose comptable, de sorte que, quand əš-Šayh Muhamd əl-Ṃâmi cherche, dans un poème en hassāniyya, à estimer le volume d'ensemble du globe terrestre, c'est bien en nombre de grains de sable qu'il propose de l'évaluer². 
Élément constitutif du désert chaud, le sable est associé à l'idée de sécheresse de l'air et d'aridité du sol, mais il arrive que des précipitations tombent, parfois même avec brutalité. D'une très bonne pluie, on peut dire as-shâb lgât at-tra (litt. « la pluie a atteint la couche humide »), dans la mesure où l'eau s'est infiltrée sous la surface jusqu'à atteindre les traces de la dernière bonne pluie. tra est un terme qui, là encore, est très proche de son équivalent classique taran « humidité; couche de terre un peu humide», mais il a tendance en hassāniyya à s'employer plus généralement dans le sens de « sable mouillé, frais, humide $»^{3}$. Le verbe dérivé de forme causative tarra s'est d'ailleurs lui aussi spécialisé: plutôt qu'« humecter quelque chose », ou même « humecter le sable », il a pris le sens particulier - encore plus étroitement lié à l'élément "sable" - de "sabler une peau avec du sable mouillé », semble-t-il pour l'assouplir.

Le sable n'est pas seulement sec ou humide, il est aussi mou ou dur. Ainsi la țaṛha correspond-elle à un espace plat et sablonneux, suffisamment uni pour être facile à la marche, tandis que (trâab) dəhsä désigne une étendue de sable mou. Cependant, le sable très mou, en région de dunes vives, porte le nom spécifique de ${ }^{\uparrow} a z^{\uparrow} a z^{4}$. Cette distinction témoigne, s'il en était nécessaire, de l'existence de différentes sortes de dunes, différence qu'il me faut maintenant examiner de plus près.

\section{Formes et structures des dunes}

Bloc diagramme. Terminologie géographique

Dans l'ensemble des termes géographiques en usage en ḥassāniyya, ceux qui se rapportent aux dunes occupent une place de choix.

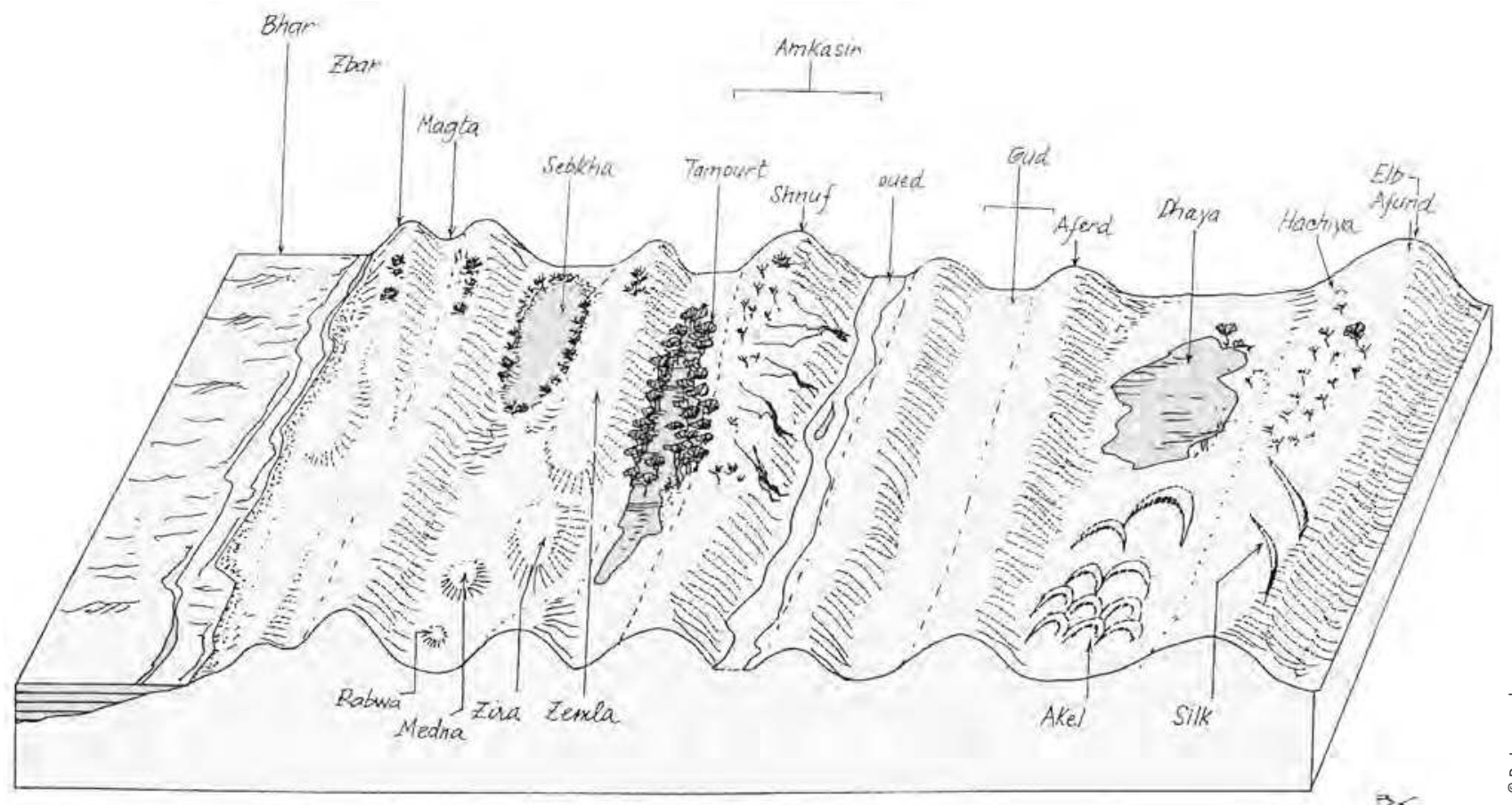


Ce qui a frappé les premiers lexicologues "hassanisants", c'est la variation des appellations en fonction de la hauteur et de la longueur. Ainsi Pierret (1948 : 140) a-t-il relevé une série de dénominations relatives aux dimensions des dunes - « très petite » (nebkè), «petite » (zīrè), « moyenne » (nebbiè), « haute mais peu allongée» (alāg), « haute et allongée » (' $\mathrm{erg}$ ), « très haute et très allongée » ('elb) - et seulement trois dénominations basées sur d'autres critères ( dune au sable très blanc » gerd, « chaîne de dunes » seïf, « région de dunes enchevêtrées » 'akle).

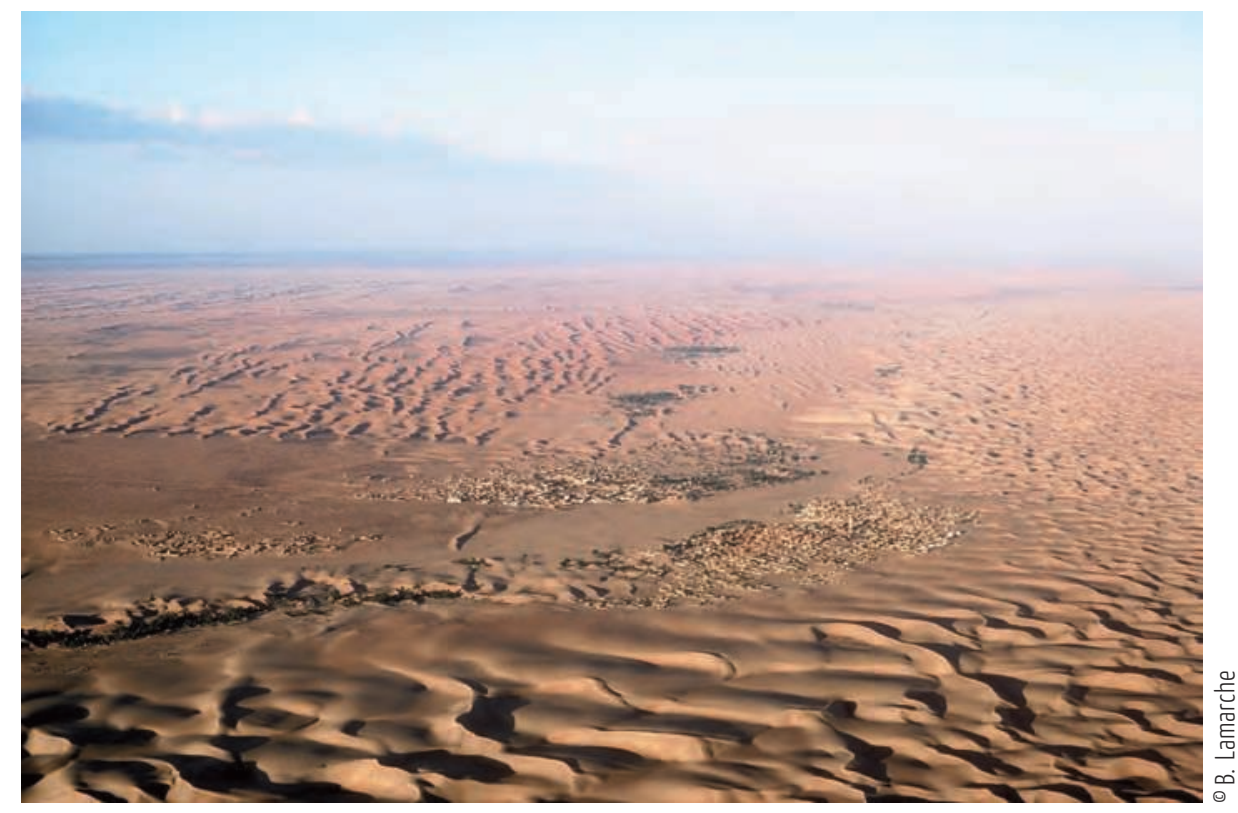

Chinguetti (vers le nord)

Est-ce en connaissance de cause, du fait de spécificités régionales, qu'il s'écarte sur plusieurs points des définitions de Reynier? Toujours est-il que la liste de mots fournie auparavant par ce dernier (1909: 106) est à la fois similaire et étonnamment divergente. $\mathrm{Si}$ `alb « dune très longue » et gourd « dune blanche, dénudée » présentent à peu près la même définition que chez Pierret, il n'en est pas de même des autres: nebka correspond cette fois à « grande dune isolée », nabia à « dune haute », zìra à " grande dune », et c'est deux nouveaux termes qui sont introduits pour désigner la « petite dune » (afound) et la « dune moyenne » (đahar). Par ailleurs, Reynier signale un autre terme, au sémantisme plus déterminé: $z b \bar{a} r a$ « dune blanche au bord de la mer $»^{5}$.

Si ces contradictions ne sont pas toujours aisées à résoudre, du moins peut-on mettre un peu d'ordre en faisant intervenir des critères plus variés. Beaucoup de dunes sont en effet nommées d'après leur forme particulière, le caractère souvent métaphorique de l'appellation facilitant alors l'interprétation. De plus, il existe une grande opposition entre « dune vive » et « dune morte », de sorte que seule une partie du lexique des dunes est indifférente à cette distinction.

Le lexème le plus englobant est en fait celui de zîrä et, en tant que terme générique (statut qu'avait reconnu Pierret), il s'applique sans doute à des dunes de hauteurs variables, 
même si l'existence du diminutif zwäyrä au sens de « butte sablonneuse » semble impliquer pour zîrä une certaine grandeur. Contrairement à ce qu'on pourrait attendre d'un terme aussi général, ce nominal n'est attesté avec ce sens qu'en hassāniyya (ses synonymes sont kațīb en arabe littéraire et ägowž en zénaga) ${ }^{6}$.

Le lexème Salb, quant à lui, est d'origine arabe, mais il n'a plus le sens de « sol dur et stérile » comme en classique. En ḥassāniyya, il désigne effectivement une dune allongée, voire très allongée, et plutôt grande (le diminutif släyb s'applique à une plus petite), mais uniquement pour les dunes fixes (dites "mortes" en français). Si `alb se distingue de `ərg, c'est moins par sa longueur et sa hauteur que par sa structure. L'appellation 'arg, à l'origine métaphorique ('́rg, comme le classique 'uraq, signifie à l'origine "veine, artère ») est en effet réservée, pour sa part, aux dunes vives.

La même opposition se retrouve dans les emplois métaphoriques de $\underline{d} r \hat{a}^{\uparrow}$ et de salk. Ces deux termes d'origine arabe, dont les sens premiers sont, respectivement, « avant-bras, coudée » et « fil (à coudre) », désignent aussi des cordons ou chaînes de dunes, mais le premier, en dunes fixes et le second, en dunes vives ${ }^{7}$. À l'inverse de ces notions de dunes régulièrement orientées, on trouve celle de ${ }^{\varsigma} a k l a ̈$ qui désigne une masse confuse de dunes vives. Il s'agit d'un terme d'origine arabe (« sables amoncelés » est l'un des sens de `awkal en classique), d'usage bien spécifique, alors qu'une dune disposée en travers d'une piste ou d'une vallée prendra un nom plus général ('arṛâț « obstacle » ou g $l i \hat{g}$ « barrage »).

L'enchevêtrement rend la traversée des dunes vives très malaisée, mais la texture du sable est une autre caractéristique qui concourt aussi fréquemment à entraver la marche. On appelle baqqârä ou bagîarä une dune de sable très mou, mais surtout ġard - un terme que l'on retrouve en zénaga (ainsi que chez les Marazig du Sud tunisien) et qui, en ḥassāniyya, désigne une dune mouvante de sable très blanc et mou ${ }^{8}$. Sur la même racine, et comme pour zîrä, le dialecte maure a créé des collectifs à préfixe ām-/am- (emprunté au berbère) : āmzîr/āmzäyyär « grand ensemble de dunes (de type zîrä)», āmigarräd " grand nombre de dunes vives (de type ġard) ${ }^{9}$.

\section{sAklat la-Mġālîg avec personnage (Théodore Monod)}

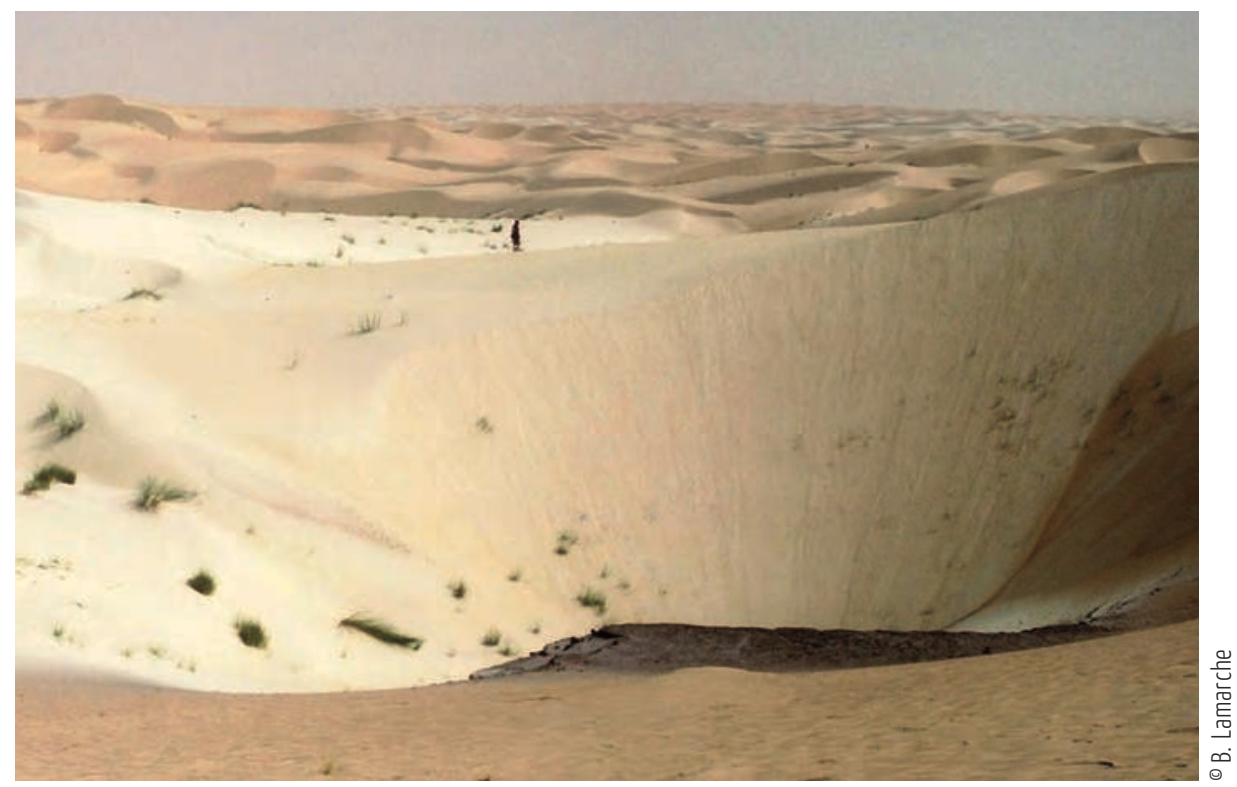


Si l'enchevêtrement est propre aux dunes vives, l'isolement est plutôt réservé aux dunes fixes, mais il se décline sur plusieurs modes.

Alors que ävärd et dämg்a désignent l'un et l'autre une dune fixe, dominante ou isolée, la définition de ävärd comporte le sème « en forme de plateau » et celle de dämg்a, celui de « à sommet arrondi ». Lorsqu'elle est à sommet pointu, la dune, comme la montagne, devient par métaphore une plume (rîšä), mais s'il s'agit en outre d'une dune dominante ou isolée, on pourra la désigner par le terme märgäb (par spécification de l'arabe marqab qui s'applique, lui, à « toute hauteur où l'on se place pour voir de loin et observer ${ }^{10}{ }^{10}$. Quant au terme a àzgi, sa définition varie beaucoup d'un auteur à l'autre (Taine-Cheikh 1988-1998: 901), l'un en faisant un " grand gard », une « dune de taille imposante et dominant les autres dans les ${ }^{\complement} a k l \ddot{a} »$, tandis qu'un autre y voit une « grande dune fixe, isolée, au sommet pointu ». Ce pourrait sembler la preuve que, en l'absence d'étymon connu, les changements sémantiques ne sont pas rares, surtout lorsque les enquêtes sont menées dans des régions très distantes. Pourtant on peut trouver chez un même auteur plusieurs définitions pour un même lexème, tel le nom nəbkä que, malgré son étymon arabe, Leriche (1955: 54) définit à la fois comme « dune haute, isolée, ronde, de sable dur » et comme « petite dune qui se forme derrière un obstacle». Mais là encore, le problème pourrait venir de l'identification du sème dominant, à chercher du côté de la nature du sable et de la forme de la pente plutôt que du côté de la hauteur de la dune.

\section{L'action du vent}

Le vent n'est pas, selon les scientifiques, le premier responsable de la physionomie des déserts, mais les actions éoliennes semblent d'autant plus efficaces dans les zones arides que leurs effets se voient très souvent à l'œil nu:

Peut-être pourrait-on dire que si c'est l'eau qui fait - ou qui a fait - l'essentiel, le travail de base: érosion, libération des sables, creusement des vallées, etc., c'est le vent qui va « fignoler » le tableau, s'occuper des détails mineurs et épidermiques: corrosions, polissages, vannages, modelages de surface dans les nappes sableuses ou les dunes, etc. (Monod, op. cit. : 56)

En hạssāniyya, le nom générique pour « vent » est rîhh (ou riyyâhh), comme en arabe classique, mais d'autres noms sont également usités en fonction des circonstances.

Certains vents sont espérés et attendus, soit parce qu'ils apportent la fraîcheur (tels rwâh « petite brise qui apporte la fraîcheur », en particulier le soir, ou ägäyâv « vent frais »), soit parce qu'ils annoncent la pluie ou l'apportent (ainsi rìh al-kôri, litt. « vent du Noir », qui vient du sud). D'autres sont redoutés, tel l'harmattan irîvi - vent brûlant qui souffle du nord-est, en particulier durant les mois de mai et de juin. Il arrive aussi que des vents portent le même nom mais soient diversement appréciés selon les régions, tel šargiyyä qui, pour les gens de l'Est et du Nord, est un bon vent, annonciateur de pluie, qui souffle au début de l'hivernage, alors que, pour ceux du Sud-Ouest, c'est un vent d'harmattan, ou encore as-sāhliyyä qui, pour les seconds, est une brise maritime, un vent d'ouest alors que pour les premiers, c'est un vent du nord ${ }^{11}$.

On remarquera que ces adjectifs nominalisés à suffixe -iyyä (əš-šargiyyä, əs-sāhliyyä) sont à la forme féminine. On peut mettre ce fait en rapport avec le cas du nom rîh luimême qui, malgré l'absence de terminaison - $a$, est resté de genre féminin comme en arabe ancien ${ }^{12}$. Ainsi accordera-t-on au féminin l'adjectif $m$ tîn « fort » dans rîh $m$ tîn-ä ou le verbe gâm «se lever » dans ər-rîh gâm-ət. 
Si la "féminisation" des lexèmes nommant des éléments naturels relève d'une attitude quasi magique de minoration - une manière de les rendre moins dangereux -, il apparaît que, pour ce qui est des vents de sable, le genre des noms est variable, mais les plus violents sont souvent de genre féminin. Ainsi sâvi « petit vent de sable » est-il du masculin, (comme en Ḥaḍramoût ou à Takroûna, cf. Landberg 1901 : 609, Marçais \& Guîga 19581961 : 1824) ${ }^{13}$, de même qu'ahattay « vent de sable », un nominal à préfixe berbère dont l'origine arabe est pourtant évidente (cf. ḥassāniyya $h \underline{h} \underline{a}$ « jeter du sable, de la poussière (sur)», apparenté au classique ḥațā « être dispersé (pour la terre, la poussière), etc. »).

Ces termes sont cependant moins usités que celui de gabra, qui signifie littéralement "poussière » (comme le classique ġabara/gubar), mais est couramment employé avec le sens de « vent de sable », alors que (a) ġmâm et qabu « brume» restent associés à la notion de «temps bouché à cause de la poussière ».

Quant aux noms donnés aux tourbillons de sable ('mâyä, za $\partial^{\uparrow} v a ̈ y g a ̈, ~ z a ̈ w b b^{\varsigma} a$ ), ils sont, comme gabra, de genre féminin. Une devinette en donne une description métaphorique: nâgt-i nâgat owlâd mbârakldarwat-hä va s-smä t'ârak/u bûț-ha va trâhb i'addäl/ la-mbârak/l

« Ma chamelle est celle des Owlâd Mbârak/sa bosse au ciel se débat/ tandis que son nombril creuse dans le sol/ des tranchées $/ / \gg^{14}$

La devinette portant sur rahž « tempête de sable » est très semblable à la précédente, mais comme le nom-réponse (rare) est du masculin, l'animal emblématique est cette fois un chameau:

žäml-i l-așfaṛ/bü-gäyd mgașsar/rașșav lo-bhaṛ/wa dägdäg la-kșaṛ/l

« Mon chameau roux/ à l'entrave courte/ peut sauter la mer/ et briser le village// » Ces devinettes, recueillies par Ould Cheikh Ahmed (1982), soulignent la puissance de certains vents de sable. Point n'est besoin cependant de vents violents, susceptibles de transporter sable ou poussière à des centaines de kilomètres ou de déplacer une dune de plusieurs (dizaines de) mètres, pour que l'action éolienne ait des conséquences sur le paysage.

Même fixes, les dunes portent l'empreinte de la direction du vent: leurs versants au vent et sous le vent ne présentent pas la même pente. Quant aux dunes vives, encore plus sensibles à l'action du vent, elles ne cessent de se remodeler au moindre changement ${ }^{15}$. Les différentes appellations en usage en ḥassāniyya pour nommer, métaphoriquement ou non, les parties de la dune, témoignent de l'intérêt que les hassanophones ont porté à la forme des dunes. Le fait que les noms d'origine métaphorique renvoient souvent à des parties du corps (ṛ̂ṣ̣ « tête », hašmm « museau effilé », šännûvä « gros nez, nez tordu », zərr " côté », 'argûb « jarret », etc.) est une manière d'assimiler la dune à un être vivant mais, sauf exception (tel l'emploi de ägâyä « couronne de cheveux tressés (des femmes) ${ }^{16}$ ), ce sont des métaphores qui s’appliquent tout aussi bien à la montagne.

\section{Le sable et le vivant}

Les paysages de sable donnent souvent l'impression d'un milieu vierge, exempt de toute présence animale ou humaine - c'est d'ailleurs là l'un de leurs charmes. La vie pourtant 
n'y est pas absente. Elle s'y fait seulement plus discrète et moins exigeante, pour s'adapter à la dureté des conditions imposées par le milieu. Mais survivre dans un tel biotope suppose aussi, pour une bonne part, savoir se repérer et apprendre à lire sur le sable comme dans un livre.

\section{Le monde végétal}

Si la plupart des espèces ne survivent pas en milieu saharien, d'autres - plantes ou animaux - ne peuvent se rencontrer ailleurs.
Groupe chamelier

abordant une zone de `aklä

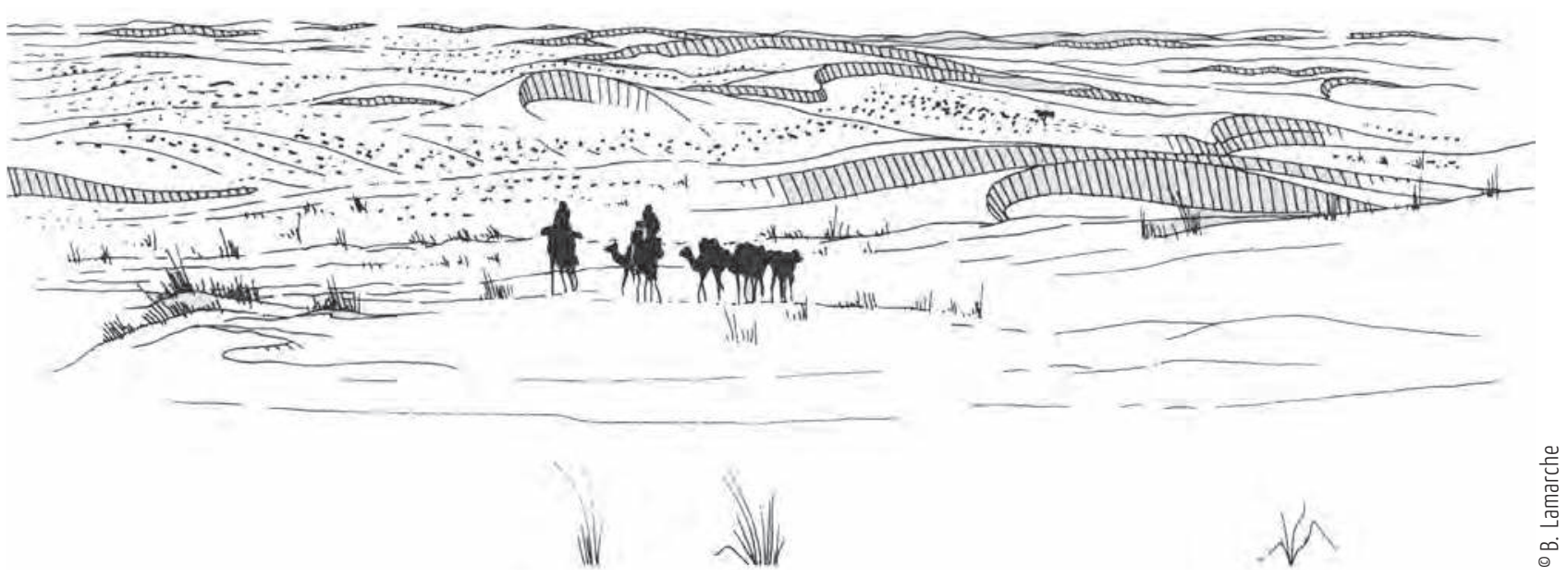

Parmi les plantes du désert, il existe aussi bien des graminées comme (um)m-rọkbä (litt. « celle au genou », Panicum turgidum) et tīzzît (Aristida funiculata) que des arbres comme ot-talh (Acacia raddiana) ou st-tämât (Acacia seyal) ${ }^{17}$. Les épineux sont nombreux. C'est le cas, non seulement des acacias, mais aussi de l'arbre täyšst (Balanites aegyptiaca) ou de l'arbuste hâd (Cornulaca monocantha) dont les feuilles charnues peuvent rester vertes trois ans après les dernières pluies. On trouve aussi des plantes à latex, telle l'euphorbe ävarnân (Euphorbia balsamifera) ou la fausse euphorbe (n)tûržä (Calotropis procera) qui atteint parfois une très grande taille. Certaines poussent sur les dunes, d'autres dans les oueds ensablés, sur les regs, sablonneux ou non, ou encore dans l'escarpement des massifs, mais elles se concentrent surtout dans les zones à fort ruissellement ${ }^{19}$.

Les plantes sont connues des nomades pour des raisons très diverses, parfois devenues proverbiales. Ainsi l'euphorbe gəndvär (Jatropha chevalieri) est-elle célèbre pour sa mauvaise odeur et la coloquinte, pour son amertume, comme en attestent les expressions suivantes: ahnäz mən gəndvär « qui sent plus mauvais que le gəndvär» et ämarr mən al-ḥanđal «plus amer que la coloquinte ». L'héliotrope ḥbāliyyä (Héliotropium undulatum ou bacciferum), plante très belle mais non comestible, est devenu le parangon de la beauté inutile (zäyn ḥbāliyyat ad-dâr « la beauté de l'héliotrope à baies ») ${ }^{20}$. Quant aux 


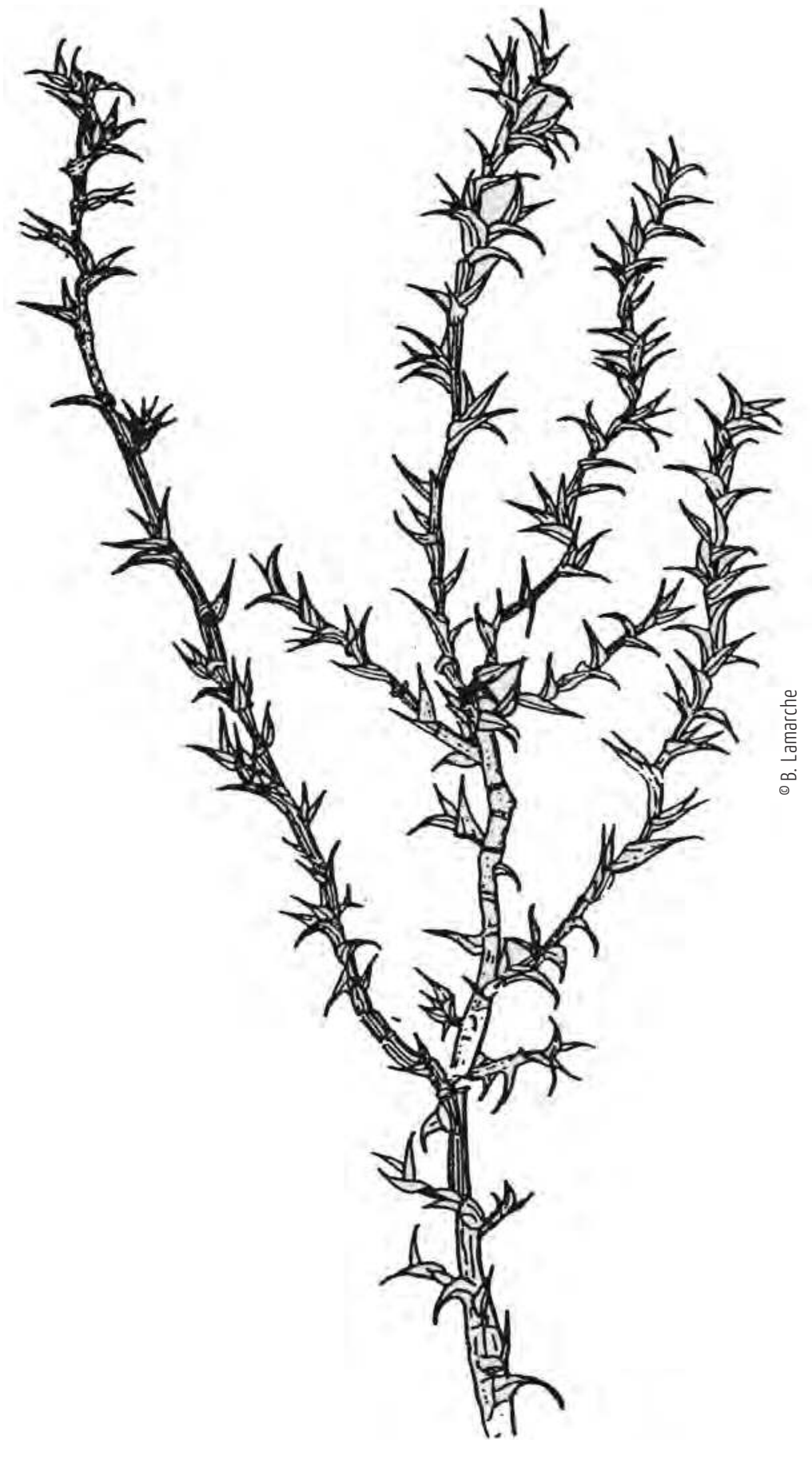

Cornulaca monacantha : hẩ euphorbes, elles sont évoquées dans plusieurs expressions pour leur fragilité. Ainsi dira-t-on de quelqu'un mal défendu qu'il a en main un « bâton de (fausse) euphorbe »

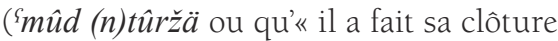
avec des euphorbes » (zarrạab b ävarnân).

Parmi toutes les plantes, beaucoup jouent un rôle important dans le biotope saharien et, faute de pouvoir les étudier une à une, je n'évoquerai ici plus en détail que le cas de la plante appelée as-sbatt/aș-ṣbat (avec ou sans emphase de la sifflante) qui a la particularité de se maintenir constamment à la surface des dunes, comme si elle nageait sur le sable. Cette graminée (Stipagrostis pungens/Aristida pungens) peut en effet être considérée comme emblématique de la zone saharienne, tout comme la graminée inîti (Cenchrus biflorus), peut l'être de la zone sahélienne ${ }^{21}$.

Le sbat est une grande graminée vivace à feuilles dures, dont le nom semble venir de la racine arabe $s-b-t$ : sabt « plat - pour des cheveux non crépus ». Il est très recherché par le bétail au printemps, quand il verdit ou fleurit. Ses fleurs, qui portent le nom de ìlîg (<zénaga äyyigi) sont réputées très favorables à la lactation des chamelles. Lorsque les feuilles sont encore un peu vertes, elles portent le nom berbère d'azâarân, mais lorsque la plante est tout à fait sèche, elle se transforme en paille. Cette paille, nommée halvä (terme d'origine arabe qui a donné le français alfa), sert alors aussi bien à nourrir les animaux qu'à confectionner toutes sortes d'objets utiles aux nomades, des cordes aux couvre-pis des chamelles.

\section{Le monde animal}

Du point de vue de son utilité pour l'homme, l'animal du désert est, par excellence, le chameau (ou plus précisément, 


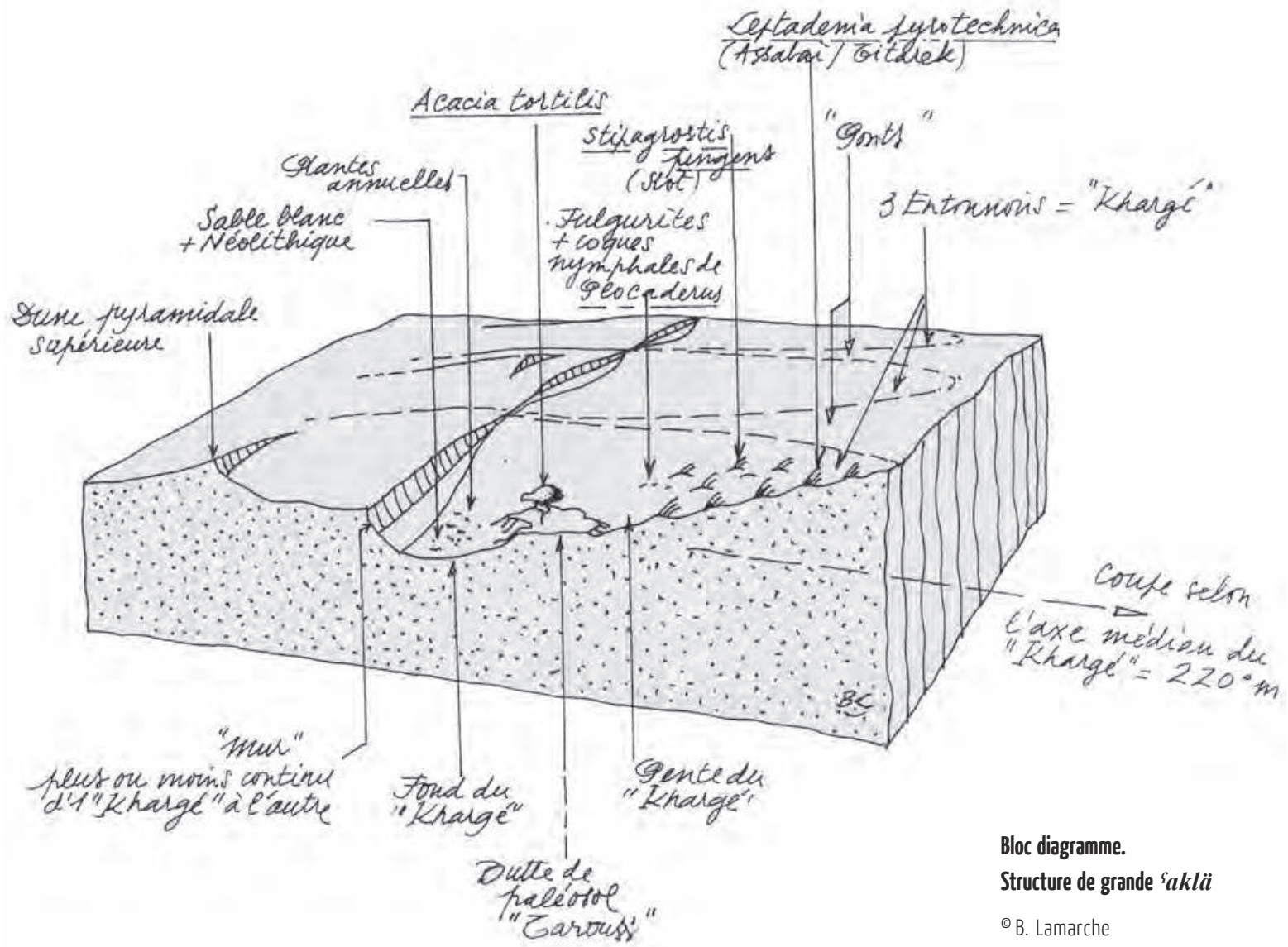

s'agissant de l'Afrique, le dromadaire - en ḥassāniyya ibal pour le générique, əž-žmäl pour le mâle, an-nâgä pour la femelle) ${ }^{22}$. Physiologiquement, le chameau est fait pour résister à la chaleur et à l'absence prolongée d'abreuvoir. Quand viennent les premières pluies, il sent de très loin les promesses de pâturage et fera tout, même entravé au plus près, pour prendre le large en se dirigeant vers elles. Mais son appétence pour toutes sortes de plantes du désert et sa capacité à se «faire une bosse » pour les périodes ou les régions les moins arrosées lui permettent de vivre et de faire vivre les nomades dans un milieu globalement fort hostile ${ }^{23}$. À cela, on peut encore ajouter au moins deux caractéristiques qui témoignent d'une indiscutable adaptation à la vie dans le désert, d'une part ses larges sabots qui lui facilitent la progression dans le sable (même si les dunes très meubles ne sont pas sans gêner sa marche), d'autre part son système respiratoire doté d'une sorte de clapet anti-retour qui lui permet de limiter la pénétration du sable dans ses naseaux en cas de tempête) (voir l'article de B. Faye dans ce numéro).

Si les bovins (bgar) sont cantonnés à la zone sahélienne, il n'en va pas de même des ovins-caprins ( $\dot{g} n \ddot{a} m$ ), mais ceux-ci n'ont quand même pas les mêmes capacités d'adaptation au désert que le chameau. Cependant, hors de la sphère domestique, au 


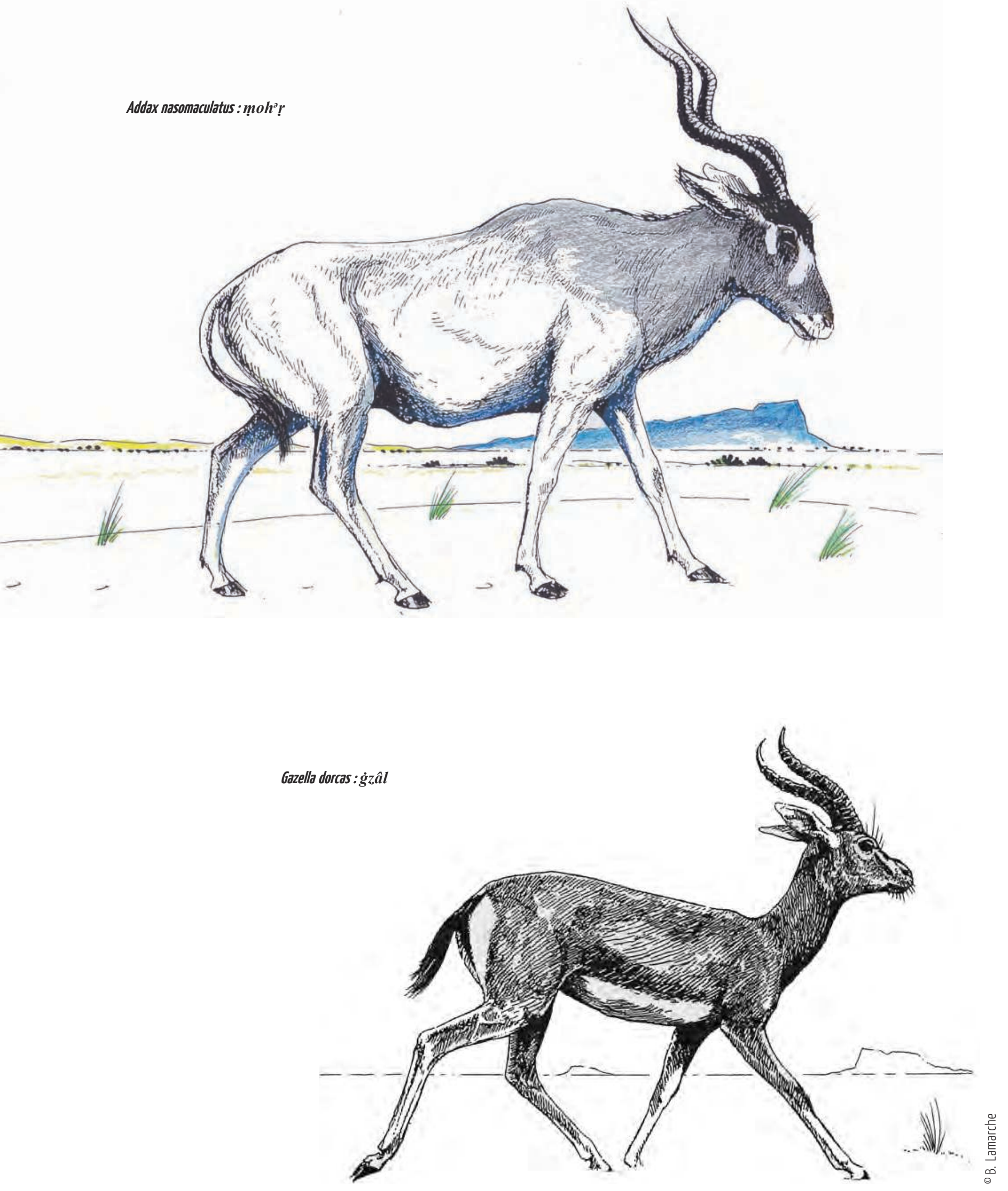


demeurant fort restreinte, on trouve une faune bien plus diversifiée qui, pendant des siècles, a survécu (presque) sans problème au Sahara.

Plusieurs espèces ont disparu de la région au cours du xx $x^{e}$ siècle, essentiellement $\mathrm{du}$ fait des hommes, tels le mouflon à manchettes (ar-râwi), l'oreillard ou Cynhyène (kälb la-hlä litt. «chien de la brousse ») et peut-être même l'antilope Addax (la-mhä), pourtant encore bien représentée, il y a peu, dans les grandes étendues désertiques de l'extrême Nord: ne dit-on pas d'une rencontre improbable, qu'elle est tfâg 'anz la-mhä u 'arr « (comme) la compagnie de l'Addax et du phacochère », chacun représentant le Sahara et le Sahel extrêmes ${ }^{24}$. Mais la disparition la plus emblématique est sans doute

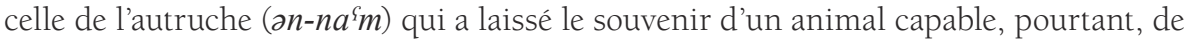
très grands déplacements, comme en témoigne l'expression proverbiale äläwwäd man varg $n^{\varsigma} a ̂ m$ « plus vagabond qu'un troupeau d'autruches ».

De toutes les espèces qui ont survécu, s'il y en a une qui incarne particulièrement le désert et rivalise peut-être avec le chameau pour ses capacités d'adaptation au Sahara, c'est la gazelle dorcade (appelée lo-ġzâl en hassāniyya et äžänkự̛ en zénaga). Plusieurs proverbes la prennent en exemple, notamment celui, très connu, qui fait de cet animal le symbole de la fidélité à sa terre d'origine: la- $\dot{g} z \hat{a} l$ (lli) imût $ə v$ žadb-u « la gazelle (qui) meurt dans son espace aride ». La plupart des nomades maures sont très sensibles au comportement de la gazelle, réputée ne pas changer de lieu, même s'il n'y a plus d'eau. Moins connues et presque énigmatiques sont, par contre, les expressions portant sur le comportement «psychologique » de la gazelle dorcade Gazella dorcas (ädärdäm mon 'anz gazlân «Plus "mère-poule" qu'une femelle gazelle ») ou de sa cousine, la gazelle à front roux Gazella rufifrons (ahallä man dāmiyyä « Plus divorceur qu'une gazelle à front roux ») ${ }^{25}$.

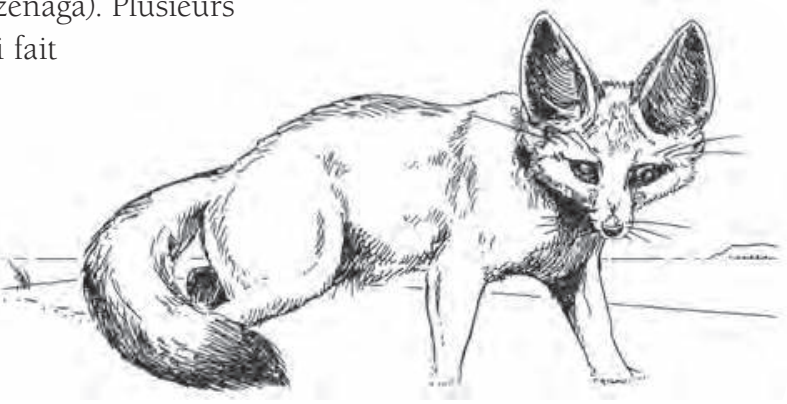

Parmi les autres animaux du désert, les moins sympathiques occupent souvent une place prépondérante dans les proverbes. Les serpents tendent à susciter une crainte dérai-

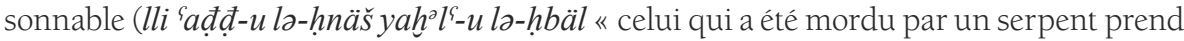
peur de la corde »), mais ils représentent le comble de la dangerosité quand ils sont excités par la chaleur (on dit d'une personne très nuisible qu'elle est comme hänš al-gâylä «le serpent de midi ${ }^{26}{ }^{26}$. Les chacals sont considérés comme des voleurs de bétail, aussi dit-on notamment d'une chose incroyable qu'elle est tmâbi ađ-đîb 'an sarhhət lo-ġnäm « (comme) le refus du chacal de garder les ovins-caprins ». Quant aux différentes variétés d'hyènes, tachetées ou rayées, elles apparaissent à bien des égards comme la méchanceté incarnée, d'où l'expression proverbiale: l-umm māmûnä yälu kânat gābûnä « la mère est digne de confiance, même si c'est une hyène » (quand le danger, ou le mauvais exemple, vient

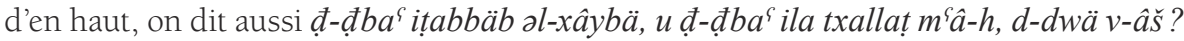
« l'hyène guérit du charbon; mais si c'est elle qui a le charbon, comment la guérir? » ${ }^{27}$ ).

Tous ces animaux sont, avec le lièvre (ən-näyräb) et le hérisson (al-gänvûd) ${ }^{28}$, des héros récurrents des contes et ils ont tendance à faire oublier d'autres espèces

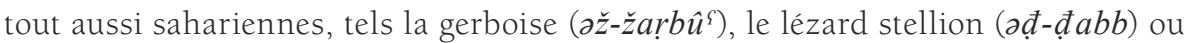
le fennec. Cette dernière espèce, rendue célèbre par Saint-Exupéry (Le petit prince, 1943), pourrait cependant paraître plus emblématique du désert que le chacal. En effet, la première porte un nom d'origine berbère (ḥassāniyya $\bar{a} \dot{g} \partial r \grave{s} i$, par emprunt au zénaga äğaršäy) alors que le chacal, qui endosse le rôle du renard Goupil dans les contes européens, porte chez les Maures un nom arabe (hassāniyya əđđ-đîb, peut-être 
à l'origine du zénaga äżđīh). Il faut noter que ce terme, à l'origine, désigne le loup animal, il est vrai, inconnu sous ces latitudes.

\section{Les hommes du désert}

La poésie strophique des Maures (lo-g்nä), souvent géographique, abonde en toponymes. Elle témoigne fréquemment d'un double attachement du poète, à la fois pour une femme aimée (ou simplement désirée) et pour les lieux où la rencontre s'est produite. Palmeraies et puits font partie des endroits les plus propices à de tels faits, mais les vers, qui décrivent aussi les trajets pour s'y rendre, évoquent en général les reliefs les plus remarquables (vallon, petite colline, piton rocheux...) et les plus beaux souvenirs de pâturage (cf. Taine-Cheikh 2006). Si le terme ar-rîm, qui signifie « gazelle» en arabe classique, est un des vocables les plus courants pour évoquer la bien-aimée - établissant ainsi un lien assez étroit entre l'imaginaire poétique maure et le monde saharien-, les topographies dunaires n'occupent pas une place très importante dans le g $\dot{g} \ddot{a}^{29}$. Voici toutefois un joli contre-exemple, dû au grand poète Mḥammäd wəll Aḥmäd Yūṛa (Ould Ahmedou Bamba 1981 : 27-28):

gäyyäm-l-i məošrog wa đ̣wäyyât $w$-al-\{alb alli vî-h at-tämât huwwä gâa ämtän-hä läy $\hat{y}^{\uparrow} \hat{t}$ [u] lukäyr amn ar-raff ab-dahlî-h

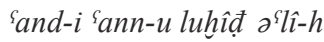

« Ils ont éveillé en moi, Mechrag et Đ̣wayyât/ el-Bawl, de bien forts tourments, «Et la dune où poussent les acacias / Cette dune qui abrite 1-Aṛbât sous son aile, «C'est elle vraiment qui me tourmente le plus. / Insolente qu'elle est! ${ }^{30}$ « Mais Loukeyr est plein d'amour secret, / Et je crois que quiconque possède un fond d'amour, «Plutôt que d'y passer,/ Ferait mieux de le contourner. »

Comme tous les vrais habitants du désert, les Maures n'ont pas vraiment choisi de vivre dans des conditions aussi difficiles. Ils n'avaient pas, comme certaines des espèces végétales ou animales évoquées plus haut, de véritables prédispositions physiologiques pour faciliter leur survie. Le sable reste donc pour eux, dans la plupart des circonstances, moins un allié qu'un élément hostile avec lequel il faut compter. Au fil des siècles, cependant, ces nomades ont développé des savoir-faire qui leur ont permis de s'adapter et même de tirer parti au mieux de l'environnement saharien, de ce qui y pousse et de ce qui y vit.

Ils ont adopté des vêtements (le turban hawli des hommes et le voile mälọfä des femmes) les protégeant du soleil comme des vents de sable. Ils ont appris à cuire la viande dans un trou (həvrạ) de sable, en se servant d'un grand bâton (äšäwwây) pour pousser les braises et retourner la viande. Dans le Nord, en période de grande abondance, certains ont même su faire du lait séché (gâras) dans des trous creusés dans le sable. Dans l'agriculture, on a employé du sable pour fumer le sol ( gäylä). Les artisans ont découvert la vertu du sable pour décaper l'or et l'argent (šäbbäb). Quant aux éleveurs, il leur est arrivé de prendre du sable pour certains soins, tel celui de la cataracte du chameau.

Les nomades savent qu'ils peuvent, en cas de nécessité, remplacer l'eau et faire leurs ablutions au sable (täyämmum), mais le sable n'est pas pour eux qu'un pis-aller. Beaucoup de jeux de société traditionnels nécessitent un espace de sable fin. C'est le cas des jeux 
de damiers, à vingt ou quarante cases (hräybgä ou đ̣̂amət/șrand). C'est plus encore celui du jeu de sîg - jeu de baguettes constituant une variété locale de jonchet - pour lequel on prépare spécialement le terrain (barṛa as-sîg) en formant une petite dune de sable au sommet aplati. Enfin, le sable est le lieu par excellence où se pratique la divination (lo-gzânä) qui, chez les Maures, se fait principalement à l'aide de traits dans le sable (d'où l'appellation de hatt ar-raml(ä), litt. «traits du sable ») ${ }^{31}$. Pour le non initié, ces traits resteront bien confus, comme le souligne la devinette qui prétend dépeindre la divination:

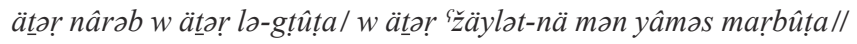

« Traces de lapins et traces de chats (sauvages)/ et traces de notre génisse depuis hier attachée $/ / »^{32}$

Si ces traces ont besoin d'une personne versée dans leur interprétation, il n'en est pas de même des autres traces observables dans la nature. Les nomades étaient en effet invités dès leur plus jeune âge à étudier les différentes empreintes laissées sur le sol et, plus largement, à porter attention à tous les indices inscrits dans le paysage (voir l'article de L. Gagnol dans ce numéro).

Les éleveurs devaient pouvoir reconnaître les animaux à leurs traces, pour les rattraper quand ils s'étaient éloignés (parfois malgré leurs entraves), pour retrouver la bête égarée ou volée, pour identifier une bête étrangère au troupeau. Les « devinettes pastorales » (az-zärg) rendent bien compte du don d'observation des bergers et de leur savoir, acquis au fil des années, qui permet aux meilleurs d'entre eux de reconnaître aussi bien la trace du chameau borgne (au fait qu'il ne mange les feuilles que d'un côté de l'arbre) que celle du chameau blanc ou pie à pattes blanches (au fait que la bête « piétine ses poils » (z-zâylä lli) täwța va šsar-ha $)^{33}$.

Lobservation des traces des animaux est également très importante pour les chasseurs et les Nmâdi qui, en tant que chasseurs quasi professionnels, ont évidemment une réputation d'excellents pisteurs. Cependant, pour eux plus encore que pour le reste de la population maure, la connaissance des traces est d'abord une question de survie, dans la mesure où ils viv(ai)ent jusqu'à récemment dans des régions particulièrement désertes et peu fréquentées. Dans La Fille du chasseur, l'héroïne explique pourquoi sa mère lui avait appris, toute petite, à reconnaître ses traces dans le sable et comment cela lui avait permis, des années plus tard, de retrouver son fils perdu sur une plage d'Espagne (Caratini 2011 : 60-61).

Plus que d'autres, ceux qui, comme les Nmâdi, partent pour de grandes marches dans le désert en solitaire ont besoin d'un sens de l'orientation très développé. Mais savoir s'orienter (stänyär ${ }^{34}$ ) est, chez le nomade, une nécessité de chaque jour, ne seraitce que pour pouvoir incliner son corps dans la bonne direction pendant la prière. Dans l'environnement quotidien, telle dune ou tel arbre peut suffire à indiquer la direction de La Mecque, de même que, pour les déplacements en terrains connus, tout repère visible (tout $a \check{s} \ddot{a} y v$ ) sert à donner la route. Mais plus généralement, et surtout pour des traversées moins balisées, l'orientation se fait en fonction des points cardinaux ou, plus précisément, de la rose des vents. En effet, tandis que les vents dominants impriment leur empreinte dans la forme des dunes - et jusque dans les plus petites ondulations sablonneuses -, une identification se fait entre le dos de la dune ou le dos de la tente d'une part (la-gvä «le dos ») et une des quatre directions cardinales (täll mais gâvä « aller vers le täll») d'autre part - direction qui a la particularité de varier un peu en suivant l'orientation du vent dominant, et correspond donc, selon les régions, tantôt au nord, tantôt à l'est.

On trouvera ailleurs (Taine-Cheikh 1991) plus de détails sur le rôle de la tente dans la rotation de la rose des vents, mais il suffit ici de relever que c'est le jeu du vent sur le sable qui fournit au nomade quelques-uns des repères essentiels à son orientation... quand ce ne sont pas les seuls! 
Le nomade, qu'il garde son troupeau, chasse la gazelle, poursuit un rezzou ou simplement se déplace d'un point à un autre, aura toujours à cœur d'observer son environnement et de chercher à décrypter les signes perçus. Ces signes peuvent être sonores, mais ils sont plus fréquemment matériels, car le sable, milieu particulièrement réceptif aux influences extérieures, tend à garder toutes les empreintes, celles du vent comme celles des êtres animés.

Dans les sociétés traditionnelles vivant en milieu désertique, notamment chez les Indiens d'Amérique et chez les Aborigènes d'Australie, on rapporte la capacité surprenante acquise par certains individus dans l'interprétation des traces. Est-elle moins développée chez les Sahariens? Est-ce seulement parce qu'ils attendent encore leur Tony Hillerman ou leur Arthur Upfield?

Il existe toutefois un indice de l'importance des traces dans la culture maure, c'est le fait que le lexème qui signifie « trace » (ätr $)$ en arabe a été grammaticalisé en hassāniyya pour donner une forme à l'expression du possible (ätṛ-u... « il est possible qu'il, peut-être qu'il... »). Le distique suivant (une təobî $\hat{\imath}^{\uparrow} a$ composée par une femme) en donne une illustration:

$W$ ätr $-\partial k m \bar{a}$ šavtî- $h$

Vowg as-sem`a yalbas tarkî- $h$

«Et peut-être que tu [femme] ne l'as pas vu

Quand il mettait sa chemise à l'étage au-dessus »

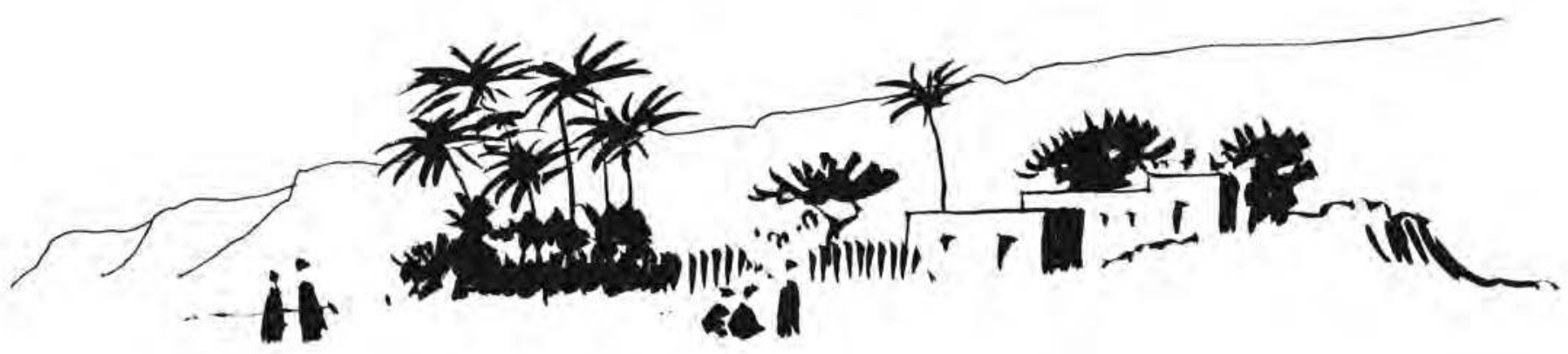

Fond d'oued ensablé

${ }^{\circ}$ B. Lamarche 


\section{NOTES}

Photo d'ouverture: Oued Timinît (vers le nord), (c) B. Lamarche.

Toutes les illustrations de cet article sont à mettre au crédit de Bruno Lamarche.

1. Il s'agit d'une variété d'arabe qui, dans certains champs lexicaux, a fait de nombreux emprunts au berbère zénaga. Je tenterai incidemment de préciser ce qu'il en est mais, sur le sujet traité ici, je n'ai pas relevé un lexique très important dans le dictionnaire de zénaga (Taine-Cheikh 2008).

2. əš-Šayh Muḥamd əl-Mâmịi (m. en 1865) est une grande figure théologique du XIX ${ }^{\mathrm{e}}$ siècle. C'est aussi un lettré et un grand poète. Sa poésie en dialectal a la particularité d'être tout à fait absconse.

3. L'argile humide porte le nom de țamza (à l'étymologie mystérieuse). En revanche, tûubä désigne une motte assez friable, c'est-à-dire un morceau d'argile, de boue séchée ou une boule de sable humide.

4. Dans la région de Tombouctou, Poussibet (op. cit.) a relevé, comme synonyme de at $t^{\uparrow} \hat{t}$, le terme fäšs $v w a \bar{s} s a$ (dont dérive probablement le français “fej-fej" employé localement par les francophones).

5. Je tiens compte ici, pour la transcription en caractères latins, de celle en caractères arabes des auteurs - imparfaite mais plus précise, notamment en ce qui concerne la longueur des voyelles.

6. En berbère, la racine $\mathrm{Z}(\mathrm{Z}) \mathrm{R}$ est celle du verbe « voir » (zénaga yäzzu? r). On peut aussi faire un rapprochement avec le touareg azyar «nuée (de poussière)» (Prasse \& al. 2003: 929) et täzyart «poussière atmosphérique » (Heath 2006 : 836), même si le sens premier est « ciel ». Cependant, l'hypothèse la plus probable est sans doute celle que m’a indiquée Lentin, selon laquelle on pourrait avoir zīra pour žazīra « île » (Dozy 1881 : I, 618 - d'après le Muhịt d'Al-Bustānī, p. 384, col. 3, sous ZWR). L'exemple est syrien, et le sens est différent de « dune », mais l'attestation du même mot avec le sens de «bouquet de palmiers » dans la région Sud tunisienne de Tozeur (Puig 2003 : 54) montre qu’un changement sémantique a déjà commencé au Maghreb pour ce mot.

7. $\Upsilon^{\prime} r^{e} g$ et $\underline{d} r \hat{a}^{\varsigma}$ ont également des emplois géographiques de type métaphorique dans l'arabe du Sud tunisien (Boris 1958 : 401, 192), mais sans référence à la distinction « dunes vives vs fixes ».
8. Selon certaines sources, elle est en forme de croissant, comme l'est la dune dite hgäf (du classique ḥiqf, de sens très proche).

9. La présence de ce préfixe pourrait être le signe que ces racines sont d'origine berbère, mais pas nécessairement en être la preuve.

10. Ce lexème arabe, avec le glissement de sens observé dans le dialecte, est le meilleur argument dont je dispose pour faire de la racine berbère « voir » la source de zîrä.

11. Je reviendrai plus loin sur ce phénomène curieux qui est lié à la question de la dénomination changeante des points cardinaux.

12. Sur ce phénomène on pourra consulter: Feghali \& Cuny (1924) pour le sémitique, Taine-Cheikh (1993 : 84-6) pour le hasasāniyya, Procházka (2004) pour l'ensemble des dialectes arabes.

13. sāfiy $\bar{a}^{2}$, en arabe classique, est une forme plus longue, mais également de genre masculin (cf. Kazimirski op. cit. : 1104).

14. Les Owlâd Mbâṛk sont une célèbre tribu guerrière de l'Est.

15. Pour un exposé didactique des rapports entre la forme des dunes et le vent, on pourra consulter l'article du lieutenant Bayard (1947).

16. Selon la définition de Poussibet (1962) : «partie saillante, promontoire - dans une dune - faisant songer à l'egâye des femmes ».

17. De nombreux noms de plante semblent d'origine berbère. Certains ont été dûment relevés en zénaga, tels

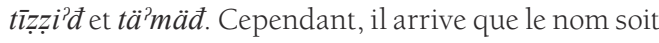
différent en hassāniyya et en zénaga, tel le Panicum turgidum qui porte le nom de $\operatorname{tgi}^{i} g a S$ dans le berbère de Mauritanie.

18. Elle est appelée tûržä ou ntûržä selon les régions. On la surnomme parfois šäyb at-trâb « les cheveux blancs de la terre » parce qu'elle tend à envahir des sols épuisés.

19. Sur les paysages végétaux et, plus généralement, sur les plantes et les animaux du Sahara, on pourra consulter avec profit la contribution de Lamarche dans Sahara. L'Adrar de Mauritanie (2002 : 158-182). 
20. Un autre proverbe se moque aussi de sa faiblesse: lo-ḥbāliyyä mā hafậtot râș-ha tahfaụ tizzîtt! « l'herbe hbāliyyä n'a pu se protéger elle-même, comment pourrait-elle protéger la plante tizzît (qui est une paille à piquants)!».

21. Cette plante (comestible pour les animaux et dont les graines sont également consommées par les hommes en période de disette) est appelée familièrement "cramcram" en français, à cause de ses nombreuses petites épines. Un journal (http://www.ar.initi.net/) a récemment adopté ce nom inîti comme titre et, pour slogan inîti yagrä $w$-idagg "Inîti colle et pique ».

22. Voir Ould Cheikh 1989, en particulier pp. 112-115.

23. En cas extrême, le chamelier pourra même faire une incision et boire "l'eau" de sa panse - opération à laquelle l'animal a cependant peu de chance de survivre.

24. Parmi les espèces sahéliennes disparues, il aurait fallu citer au moins l'éléphant ( $\partial l-v \hat{\imath} l)$, le lion $\left(\partial s-s b a^{\varsigma}\right)$ et l'antilope oryx (al-warg).

25. Cette dernière, qui semble porter en ḥassāniyya un nom d'origine berbère (on l'appelle äžəmmi en zénaga, mais édemi dans le touareg de l'Ahaggar, cf. Foucauld 1951-1952: I, 200) est considérée comme une espèce ou variété de plus grande taille. Quant à la grande gazelle dama, dite biche Robert (hassāniyya noh $^{\circ}$ ṛ/ zénaga äna?r), elle évolue exclusivement en milieu arboré, mais aurait quasiment déserté la Mauritanie.

26. Parmi les variétés connues comme réellement dangereuses, on peut citer le gammâs (litt. « le plongeur »), nommé ainsi parce qu'il a l'habitude de s'enfoncer profondément dans le sol sableux.

27. Il existe plusieurs dénominations en hassāniyya pour les hyènes. šärțât et gābûn désignent la même espèce - vraisemblablement l'hyène commune, tachetée (Crocuta crocuta), encore appelée garyâv. šärțât est usité à l'est du pays et gābûn (à rapprocher du zénaga

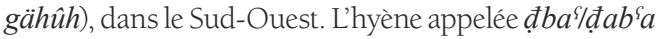
en ḥassāniyya (ärợäy en zénaga), plus petite, serait la variété rayée (Hyena hyena).

28. Le lièvre « affectionne les bonnes grosses touffes de Panicum turgidum », (um)m-ṛ əkbä, tandis que les hérissons (le hérisson du désert, Paraechinus aethiopicus), comme celui à ventre blanc, « sont tout particulièrement inféodés aux secteurs complantés de Calotropis procera », (n)tûržä (Lamarche, op. cit. : 177).

29. Ould Cheikh (2007: 10) notait, dans sa présentation à la réédition de La grande foire des dattes d'Odette du Puigaudeau (1937), la propension de l'auteure à comparer la forme des dunes aux courbes du corps féminin. Ce type de comparaison sensuelle n'est pas attesté chez les poètes maures.
30. Le sens de l'hémistiche, globalement, est dépréciatif (par antiphrase), la signification littérale d'arbât-u $w$-arbât arbât $-u$ étant « ses fermetures et les fermetures de ses fermetures $»$.

31. Pour plus de détails, on pourra se référer à l'article de Trancart (1938)

32. Il est en effet d'usage d'entraver les grands veaux pour les empêcher d'entraîner à leur suite les jeunes veaux vers les pâturages où ils rejoindraient leurs mères et les téteraient.

33. Pour plus de détails sur ces devinettes d'un genre particulier, voir Taine-Cheikh 1995.

34. Sur cette forme verbale et sur la curieuse forme causative sänyär « orienter », voir Taine-Cheikh 2003.

35. À noter qu'une grammaticalisation comparable existe dans quelques parlers orientaux de nomades (cf. Taine-Cheikh 2013: 149-151). 


\section{RÉFÉRENCES}

Bayard, Lt. 1947 Aspects principaux et consistance des dunes (Mauritanie). In Bulletin de l'IFAN série B, Tome IX, n' $1-4: 1-17$.

Boris, G. 1958 Lexique du parler arabe des Marazig. Paris: Klincksieck.

Caratini, S. 2011 La Fille du chasseur. Paris: Éditions Thierry Marchaisse.

Dozy, R. 1881 Supplément aux dictionnaires arabes. Tome I. Leyde: Brill.

Feghali, M. \& Cuny, A. 1924 Du genre grammatical en sémitique. Paris: Librairie Orientaliste P. Geuthner.

Foucauld, Ch. de 1951-1952 Dictionnaire touareg-français (Ahaggar). Paris: Imprimerie Nationale de France.

Heath, J. 2006 Dictionnaire touareg du Mali. Tamachek-anglais-français. Paris: Karthala.

Kazimirski, A. de B. 1860 Dictionnaire arabe-français, Tomes I et II. Paris: Maisonneuve et Cie.

Lamarche, B. 2002 Le Milieu. In S. Estibal, A. E. W. O. Cheikh, B. Lamarche, R. Vernet \& al. (eds), Sahara. L’Adrar de Mauritanie. Sur les traces de Théodore Monod. Paris: Vents de sable: 158-182.

Landberg, comte C. de 1901 Étude sur les dialectes de l'Arabie Méridionale. Tome I. Haḍramoût. Leiden: Brill. Leriche, A. 1955 Terminologie géographique maure. St-Louis: IFAN - Mauritanie.

Marçais, W. \& Guiga, A. 1958-1961 Textes arabes de Takroûna, Tome II. Glossaire. Paris: Librairie orientaliste P. Geuthner.

Monod, Th., en collaboration avec J.-M. Durou 1988 Déserts. Marseille: AGEP.

Ould Ahmedou Bamba, M. 1981 M'hammed Ould Ahmed Youra poète amoureux. Maîtrise de Lettres Modernes. Nouakchott: École Normale Supérieure de Nouakchott.

Ould Cheikh Ahmed, M. E.-A. 1982 Ithaži devinettes hassaniya. Maîtrise de Lettres Modernes. Nouakchott: École Normale Supérieure de Nouakchott.

Ould Cheikh, A. W. 2007 Présentation « Voyage, voyages... ». La grande foire des dattes (Adrar mauritanien) d'Odette du Puigaudeau (1937). Paris: Ibis Press: 7-15.

— 1989 Une aire de transit. In G. Courrèges, Y. de La Croix \& P. Peteuil (eds), Sud Sahara Nord Sahel. Abidjan: Centre Culturel Français Abidjan: 111-124.

Pierret, R. 1948 Étude du dialecte maure des régions sahariennes et sahéliennes de l’Afrique occidentale française. Paris: Imprimerie nationale.

Poussibet, F. 1962 Répertoire des termes géographiques de la région de Tombouctou. In Bulletin de IFAN série B, Tome XXIV, nº 1-2: 199-262.

Prasse, K.-G., Alojaly, G. \& Ghabdouane, M. 2003 Dictionnaire Touareg - Français (Niger). Copenhague: Museum Tusculanum Press - Université de Copenhague.

Procházka, S. 2004 Unmarked feminine nouns in modern Arabic dialects. In M. Haak, R. de Jong and K. Versteegh (eds.), Approaches to Arabic dialects: Collection of articles presented to Manfred Woidich on the occasion of his sixtieth birthday. Leiden - Boston: Brill: 237-262.

Puig, N. 2003 Bédouins sédentarisés et société citadine à Tozeur (Sud-ouest tunisien). Tunis-Paris: IRMC - Karthala. Reynier, A. 1909 Méthode pour létude du dialecte maure. Tunis.

Saint-Exupéry, A. de 1943 Le Petit Prince. Paris : Gallimard.

Taine-Cheikh, C. 2013 Grammaticalized uses of the verb ra(a) in Arabic: a Maghrebian specificity? In M. Lafkioui (ed.), African Arabic: Approaches to Dialectology. Berlin: Mouton De Gruyter: 121-159.

- 2008 Dictionnaire zénaga - français. Le Berbère de Mauritanie par racines dans une perspective comparative. Köln: Köppe.

— 2006 Poésies d'itinéraire et itinéraires poétiques au Sahara. In S. Naïm (dir.), La Rencontre du temps et de l'espace. Approches linguistique et anthropologique. Leuven-Paris-Dudley: Peeters (SELAF): 139-163.

- 2003 Les Valeurs du préfixe s- en hassaniyya et les conditions de sa grammaticalisation. In I. Ferrando \& J.J. Sanchez Sandoval (ed.), AIDA 5th Conference Proceedings. Cádiz: Servicio de Publicationes Universidad de Cádiz: 103-118. 
— 1995 Les Bergers maures se lancent des "colles". In Littérature Orale Arabo-Berbère no 22-23: 173-204.

- 1993 Du sexe au genre: le féminin dans le dialecte arabe de Mauritanie. In Matériaux arabes et sudarabiques, n 5 (N. S.): 67-121.

— 1991 Le Vent et le devant. De l'orientation chez les Maures. In Journal asiatique, Tome CCLXXIX, $n^{\circ} 1-2: 93-126$.

- 1988-1998 Dictionnaire Hassaniyya-Français. Paris: Geuthner.

Trancart, Lt A. 1938 Sur un procédé de divination de l'Adrar mauritanien le gzān. In Bulletin du Comité d'Études Historiques et Scientifiques de l'A.O.F. Tome XXI, nº 4: 489-498.

\section{POUR CITER CET ARTICLE}

Taine-Cheikh, C. 2014 Sur les traces des Maures, entre sable et poussière, in S. Boulay \& M.-L. Gélard, Vivre le sable! Corps, matière et sociétés, Techniques \& Culture 61 : 188-209. 


\section{RÉSUMÉ}

Sur les traces des Maures, entre sable et poussière. M'intéressant aux populations nomades maures (les Bị̂ân) qui (sur)vivent dans les régions désertiques et semi-désertiques d'Afrique du Nord-Ouest, j'explore, dans cet article, leurs représentations du désert saharien, et plus particulièrement du sable, à travers les ressources linguistiques et littéraires de leur dialecte, le ḥassāniyya. Dans la première partie, l'accent est mis sur la description des paysages à travers trois thèmes distincts. Tout d'abord, j'étudie les différents termes par lesquels on nomme le sable et mets en évidence certains glissements de sens observables entre l'arabe classique et le dialecte. Ensuite j'étudie le lexique des dunes et montre comment ce champ sémantique est structuré autour de l'opposition fondamentale entre dune "vive" et dune "morte". Enfin je m'intéresse à la question du vent (notamment du vent de sable, si fréquent dans la région) et à son action sur la forme des dunes.

La seconde partie porte sur les formes de vie se manifestant dans cet univers et sur l'interaction des hommes avec le milieu. J'étudie le monde végétal, puis le monde animal, en privilégiant, parmi les plantes et les animaux emblématiques de ce biotope, ceux qui ont une importance particulière pour les nomades. Je cite à leur propos un grand nombre de proverbes qui mettent en relief leurs caractéristiques réelles ou supposées. Enfin, après avoir évoqué la vision poétique que les Maures peuvent avoir du désert, je relève quelques formes lexicales qui révèlent les usages que cette population nomade fait du sable dans son quotidien. Soulignant la place que la lecture des traces prend dans leur (sur)vie, je montre que le terme même de trace est à l'origine de l'expression du possible.

\section{ABSTRACT}

Following the tracks of the Moors, between sand and dust. As I focus on the nomadic Moorish people (the Bịạan) who live (or rather survive) in the desertic and semi-desertic Northwest Africa, I explore in this article their representations of the Sahara desert, in particular their representations of the sand, through the linguistic and literary resources of their dialect, the Hassaniyya.

In the first part, I focus on the description of the landscape through three distinct themes. I start with a study of the various terms by which the sand is called, and highlight some observable shifts in meaning between classical Arabic and dialect. I study then the lexicon of the dunes, and how this semantic field is structured around the fundamental opposition between a dune that is "alive" and a "dead" dune. Finally, I discuss the question of the wind (especially sand storms, so common in the region) and its effect on the shape of the dunes.

In the second part, I focus on the forms of life present in this world and the interaction of the Moors with their environment. I study the emblematic plants and animals of this biotope, more specifically the ones that have a particular importance for the nomads, and quote the many proverbs emphasizing their real or perceived characteristics. Finally, after presenting the poetic vision that the Moors sometimes have about the desert, I identify some of the lexical forms revealing the use they make of sand in their daily life. I emphasize the role that reading tracks takes in their life (and survival) and show that the word "tracks" itself is at the root of the expression of possibility.

\section{MOTS CLÉS}

Nomades, Mauritanie, arabe, sable, dune, trace

\section{KEYWORDS}

Nomadic people, Mauritania, arab, sand, dune, tracks 